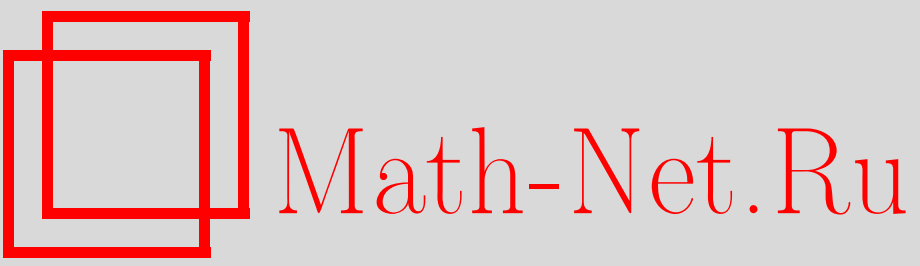

А. А. Туганбаев, Полудистрибутивные наследственные кольца, УМН, 1997, том 52, выпуск 4, 215-216

DOI: https://doi.org/10.4213/rm876

Использование Общероссийского математического портала Math-Net.Ru подразумевает, что вы прочитали и согласны с пользовательским соглашением

http://www.mathnet.ru/rus/agreement

Параметры загрузки:

IP: 18.207 .199 .55

26 апреля 2023 г., 16:40:31 


\section{ПОЛУДИСТРИБУТИВНЫЕ НАСЛЕДСТВЕННЫЕ КОЛЬЦА}

\section{А. А. ТУГАНБАЕВ}

Все колшца предполагаются ассоциативными и с ненулевой единицей. Модул $M$ назьвается дистрибутивныцм ( цепнылм), если решетка Lat $(M)$ всех его подмодулей дистрибутивна (является цепью). Прямая сумма дистрибутивных (цепных) модулей назьвается полудистрибутивныцм ( полуцепнылм) модулем. Слова типа "полудистрибутивное кольцо" означают, что соответствующие условия выполнены справа и слева. Основным результатом данной работы является

Теорема 1. Пусть $A$ - наследственное справа полусовершенное полупервичное кольчо. Равносильны условия:

(1) А - нётерово справа кольщо;

(2) $A$ - полущепное справа кольцо;

(3) $A$ - полудистрибутивное справа кольцо.

ЗАмЕчАНИЕ 1 . В связи с теоремой 1 заметим, что в ней нельзя отбросить условие полупервичности. Действительно, пусть $A$ - подалгебра алгебры всех верхнетреугольных матриц третьего порядка над полем $F$, у которой $F$-базисом служат матричные единицы $\left\{e_{11}, e_{12}, e_{13}\right.$, $\left.e_{22}, e_{33}\right\}$. По замечанию 5 работы [1] $A$ - наследственное артиново полудистрибутивное кольцо, не являюшееся полуцепным справа.

ЗАмечание 2 . Пример кольца $A$ тех рационалњных чисел, знаменатели которых не делятся на 2 и 3 , показьвает, что в теореме 1 условие полусовершенности нельзя заменить на условие полулокальности. В данном случае $A$ - нётерова дистрибутивная полулокальная область, не являющаяся полуцепным кольцом.

ЗАмечАниЕ 3 . Если $A$ - кольцо недискретного нормирования в поле, то можно проверить, что кольц $A$ дает пример того, что в теореме 1 условие наследственности нельзя заменить на условие полунаследственности. В данном случае $A$ - цепная (в частности, полусовершенная и дистрибутивная) область, не являющаяся нётеровой.

Доказательство теоремш 1 разобьем на ряд лемм. Модуль назьвается конечномерным, если он не содержит бесконечных прямых сумм ненулевых подмодулей. Подмодуль фактормодуля называется подфактором. Модуль $M$ называется счетно-дистрибутивнылм, если $M$ удовлетворяет следующим двум эквивалентным условиям: (1) никакой подфактор модуля $M$ не содержит бесконечных прямых сумм изоморфных простых модулей; (2) никакой подфактор модуля $M$ не содержит бесконечных прямых сумм изоморффных ненулевых модулей. Любой дистрибутивньй модуль $M$ не имеет подфакторов, являющихся прямыми суммами двух изоморфных ненулевых модулей, и, следовательно, $M$ - счетно-дистрибутивньй модуль. Модуль называется равномержым, если любые два его ненулевых подмодуля имеют ненулевое пересечение. Прямая сумма равномерных модулей назьвается полуравномерным модулем. Модуль назьвается риккартов $ы м$, если все его циклические подмодули проективны. Через $J(M)$ обозначается радикал Джекобсона модуля $M$. Циклический модуль $M$ назьвается локальнылм, если $M$ имеет ровно один максимальный подмодуль (т.е. если $M / J(M)$ - цепной модуль).

Лемма 1. Пусть $M-$ правыцй модуль над полулокальныцм кольцом А, причем $M$ является конечной прямой суммой счетно-дистрибутивных модулей. Тогда:

(1) все подфакторы модуля $M$ конечномерны;

(2) если $M$ - полуартинов модуль, то $M$ - артинов модуль;

(3) если $A$ - совершенное слева кольцо, то $M$ - артинов модуль;

(4) если $A$ - совершенное справа кольцо, то $M$ - нётеров модуль;

(5) если $A$ - совершенное кольцо, то $M-$ модуль, обладающий композиционным рядом.

Лемма 1 следует из леммы 15 и замечаний 1,3 и 4 работы [2]. 
Лемма 2. Пусть $A$ - полулокальное кольцо, являющееся (конечной) прямой суммой счетно-дистрибутивных правых идеалов. Тогда:

(1) $A$ - конечномерное справа кольцо;

(2) если $A$ - наследственное справа кольцо, то $A$ - нётерово справа кольцо;

(3) если $A$ - совершенное слева или справа кольцо, то $A$ - артиново справа кольцо.

(1) следует из леммы $1(1) ;(2)$ следует из (1) и того, что наследственное справа конечномерное справа кольцо является нётеровьд справа $[3,5.20]$. Докажем (2). Если $A$ совершенно слева, то по лемме 1(3) $A$ - артиново справа кольцо. Пусть $A$ совершенно справа. По лемме $1(4) A-$ нётерово справа кольцо и, кроме того, радикал $J(A)$ совершенного справа кольца $A$ является нильидеалом. Поэтому $A$ - полупримарное нётерово справа кольцо. Тогда $A$ - артиново справа кольцо.

Лемма 3. Пусть $A$ - полусовершенное кольцо, у которого все 2-порожденные правые идеаль проективны как правые $А$-модули.

Тогда $A$ - полуравномерное справа кольцо $\Longleftrightarrow A$ - полуцепное справа кольцо.

Надо доказьвать только импликацию $\Longrightarrow$. Пусть $M$ - равномерное прямое слагаемое модуля $A_{A}$. Достаточно доказать, что $M_{A}$ - цепной модуль. Допустим противное. Тогда существуют не сравнимые по включению циклические подмодули $F$ и $G$ модуля $M$. Тогда $F+G-$ 2-порожденный модуль, не являющийся локальным. Так как $F+G$-неразложимьй конечнопорожденный проективный модуль над полусовершенным колцом $A$, то $F+G$ - локальньй модуль (см. [4, 22.23]). Получено противоречие.

Лемма 4. Любое риккартово справа конечномерное справа полупервичное кольчо $A$ является полуравномерным справа.

Риккартово справа кольцо $A$ является несингулярньц справа. Конечномерное справа кольцо $A$ имеет существенньй правый идеал $M$, который является конечной прямой суммой равномерных правых идеалов $M_{1}, \ldots, M_{n}$. Так как $A$ - несингулярное справа конечномерное справа полупервичное кольцо, то $A$ является правым порядком в полупростом артиновом кольце [5, 9.13]. Поэтому существенный правый идеал $M$ кольца содержит некоторый регулярный элемент $a$. Так как $A$-риккартово справа конечномерное справа кольцо и $a A \subseteq M$, то проективный модуль $a A$ изоморфен прямой сумме некоторых подмодулей из $M_{i}$, причем $a A \cong A_{A}$. Поэтому $A$ - полуравномерное справа кольцо.

ЛЕмма 5. Любое полунаследственное справа конечномерное справа полусовершенное полупервичное кольцо $А$ является полуцепным справа кольцом.

Лемма 5 следует из лемм 3 и 4.

ДОКАЗАТЕЛЬСТво ТЕОРЕМЫ 1 . Импликация $(1) \Longrightarrow(2)$ следует из леммы 5 . Импликация $(2) \Longrightarrow(3)$ очевидна. Импликация (3) $\Longrightarrow(1)$ следует из леммы 2 (2).

\section{СПИСОК ЛИТЕРАТУРЫ}

[1] Туганбаев А. А. // Матем. сб. 1996. Т. 187. № 12. С. 137-156. [2] Туганбаев А. А. // Матем. заметки. 1995. T. 58. № 5. C. 736-761. [3] Goodearl K. R. Ring Theory. Nonsingular Rings and Modules. New York: Marcel Dekker, 1976. [4] Фейс К. Алгебра: кольца, модули и категории. Т. 2. М.: Мир, 1979. [5] Фейс К. Алгебра: колњца, модули и категории. Т. 1. М.: Мир, 1977. 